\title{
Nagumo theorems of third-order singular nonlinear boundary value problems
}

\section{Ming Cheng*}

\section{"Correspondence:}

jlumcheng@hotmail.com Institute of Applied Physics and Computational Mathematics, Beijing, 100088, P.R. China College of Mathematics, Jilin University, Changchun, 130012, P.R. China

\section{Springer}

\begin{abstract}
In this paper, we establish the Nagumo theorems for boundary value problems associated with a class of third-order singular nonlinear equations:

$\left(p(t) x^{\prime}\right)^{\prime \prime}=f\left(t, x, p(t) x^{\prime},\left(p(t) x^{\prime}\right)^{\prime}\right), \forall t \in(0,1)$ by the method of upper and lower solutions and the Schauder fixed point theorem. We also consider the multiplicity of the solutions by using topological degree theory. There are some examples to illustrate how the results of this paper can be applied.
\end{abstract}

Keywords: upper and lower solutions; Nagumo theorems; singular nonlinear differential equation; third order; Schauder fixed point theorem

\section{Introduction}

The singular differential equations arise in the fields of fluid mechanics, gas dynamics and so on. In recent years, several authors investigated the existence problems of solutions for third-order nonlinear differential equations [1-4]. The method of upper and lower solutions has become a standard tool in studying the solvability of boundary value problems associated with the differential equations [5-7]. Specially, the existence of a solution for the boundary value problem

$$
\begin{aligned}
& y^{\prime \prime \prime}=g\left(x, y, y^{\prime}, y^{\prime \prime}\right), \quad x \in(a, c), \\
& y(a)=\alpha, \quad y^{\prime}(a)=\alpha^{\prime}, \quad y^{\prime}(c)=\gamma^{\prime}, \quad a<c,
\end{aligned}
$$

is investigated in article [8]. For two-point BVPs, Yao and Feng employed the upper and lower solution method to prove the existence of solutions for a kind of third-order nonlinear differential equations [9]. For a class of third-order nonlinear boundary value problems, Du et al. used an a priori estimate, Nagumo condition, upper and lower solutions, and the Leray-Schauder degree to obtain the existence of solutions [10]. In this paper, we establish several general existence theorems for boundary value problems associated with the following singular nonlinear differential equation:

$$
\left(p(t) x^{\prime}\right)^{\prime \prime}=f\left(t, x, p(t) x^{\prime},\left(p(t) x^{\prime}\right)^{\prime}\right), \quad \forall t \in(0,1)
$$

by applying the method of lower and upper solutions, where the singularity of $p(t)$ is at $t=0$. We only consider the case of $\left.p(t)\right|_{t=0}=0$ and $p(t)>0$ on $(0,1]$. To overcome the

(c) 2015 Cheng. This article is distributed under the terms of the Creative Commons Attribution 4.0 International License (http://creativecommons.org/licenses/by/4.0/), which permits unrestricted use, distribution, and reproduction in any medium, provided you give appropriate credit to the original author(s) and the source, provide a link to the Creative Commons license, and indicate if changes were made. 
difficulty of a singularity, we give some suitable hypotheses and obtain the main result by the Schauder fixed point theorem.

Basically, the functions $\psi(t), \phi(t) \in C[0,1]$ are called upper and lower solutions of (1.1) on $[0,1]$, if $\psi^{\prime}(t), \phi^{\prime}(t) \in C(0,1), p(t) \psi^{\prime}, p(t) \phi^{\prime} \in C^{2}[0,1]$, and

$$
\begin{aligned}
& \left(p(t) \phi^{\prime}\right)^{\prime \prime} \geq f\left(t, \phi, p(t) \phi^{\prime},\left(p(t) \phi^{\prime}\right)^{\prime}\right), \\
& \left(p(t) \psi^{\prime}\right)^{\prime \prime} \leq f\left(t, \psi, p(t) \psi^{\prime},\left(p(t) \psi^{\prime}\right)^{\prime}\right), \quad \text { on }(0,1), \\
& \phi(t) \leq \psi(t), \quad p(t) \phi^{\prime}(t) \leq p(t) \psi^{\prime}(t), \quad \text { on }[0,1] .
\end{aligned}
$$

For (1.1), we adopt the following hypotheses on $p$ and $f$.

Hypothesis 1.1 $\Omega=\{x \in \mathbb{R}$; there exists $t \in[0,1]$, such that $\phi(t) \leq x \leq \psi(t)\}, \Omega_{0}=\{(t, x$, $\left.y, z) ; 0 \leq t \leq 1, \phi(t) \leq x \leq \psi(t), p(t) \phi^{\prime}(t) \leq y \leq p(t) \psi^{\prime}(t), z \in \mathbb{R}\right\}, p(t) \in C([0,1] ;[0,+\infty))$ and $\int_{0}^{1} p^{-1}(t) d t<\infty$.

Hypothesis $1.2 f \in C\left([0,1] \times \Omega \times \mathbb{R}^{2} ; \mathbb{R}\right), f(t, x, y, z)$ are nondecreasing in $x$. There exist continuous positive-valued functions $A_{i} \in C([0,1] \times \Omega ; \mathbb{R}), K_{i} \in C\left(\Omega_{0}, \mathbb{R}\right)(i=1,2)$ and $h \in C([0,+\infty) ;(0,+\infty))$, such that

(i) $|f(t, x, y, z)| \leq A_{1}(t, x) K_{1}(t, x, y, z)+A_{2}(t, x) K_{2}(t, x, y, z), \forall(t, x, y, z) \in \Omega_{0}$,

(ii) $r=\sup \left\{A_{1}(t, x) ;(t, x) \in[0,1] \times \Omega\right\}<+\infty$,

(iii) there exist positive numbers $n$ and $N$ satisfying $K_{1}(t, x, y, z) \leq|z| h(|z|)$, $K_{2}(t, x, y, z) \leq N h(|z|)$, for $|z| \geq n$ and $(t, x, y, z) \in \Omega_{0}$,

(iv) $\int_{0}^{+\infty} h^{-1}(t) d t=\infty$.

In this paper, we study the following four kinds of boundary value problems:

$$
\begin{aligned}
& \left\{\begin{array}{l}
\left(p(t) x^{\prime}\right)^{\prime \prime}=f\left(t, x, p(t) x^{\prime},\left(p(t) x^{\prime}\right)^{\prime}\right), \quad t \in(0,1), \\
x(0)=\alpha,\left.\quad p(t) x^{\prime}(t)\right|_{t=0}=\alpha^{\prime},\left.\quad p(t) x^{\prime}(t)\right|_{t=1}=\beta^{\prime},
\end{array}\right. \\
& \begin{cases}\left(p(t) x^{\prime}\right)^{\prime \prime}=f\left(t, x, p(t) x^{\prime},\left(p(t) x^{\prime}\right)^{\prime}\right), & t \in(0,1), \\
x(0)=\alpha,\left.\quad g\left(p(t) x^{\prime},\left(p(t) x^{\prime}\right)^{\prime}\right)\right|_{t=0}=0,\left.\quad p(t) x^{\prime}(t)\right|_{t=1}=\beta^{\prime},\end{cases} \\
& \begin{cases}\left(p(t) x^{\prime}\right)^{\prime \prime}=f\left(t, x, p(t) x^{\prime},\left(p(t) x^{\prime}\right)^{\prime}\right), & t \in(0,1), \\
x(0)=\alpha,\left.\quad p(t) x^{\prime}(t)\right|_{t=0}=\alpha^{\prime}, & \left.h\left(p(t) x^{\prime},\left(p(t) x^{\prime}\right)^{\prime}\right)\right|_{t=1}=0,\end{cases} \\
& \begin{cases}\left(p(t) x^{\prime}\right)^{\prime \prime}=f\left(t, x, p(t) x^{\prime},\left(p(t) x^{\prime}\right)^{\prime}\right), & t \in(0,1), \\
x(0)=\alpha,\left.\quad g\left(p(t) x^{\prime},\left(p(t) x^{\prime}\right)^{\prime}\right)\right|_{t=0}=0, & \left.h\left(p(t) x^{\prime},\left(p(t) x^{\prime}\right)^{\prime}\right)\right|_{t=1}=0 .\end{cases}
\end{aligned}
$$

Here,

$$
\begin{array}{ll}
\left.p(t) x^{\prime}\right|_{t=0}=\lim _{t \rightarrow 0^{+}} p(t) x^{\prime}, & \left.\left(p(t) x^{\prime}\right)^{\prime}\right|_{t=0}=\lim _{t \rightarrow 0^{+}}\left(p(t) x^{\prime}\right)^{\prime}, \\
\left.p(t) x^{\prime}\right|_{t=1}=\lim _{t \rightarrow 1^{-}} p(t) x^{\prime}, & \left.\left(p(t) x^{\prime}\right)^{\prime}\right|_{t=1}=\lim _{t \rightarrow 1^{-}}\left(p(t) x^{\prime}\right)^{\prime},
\end{array}
$$

$g(x, y), h(x, y)$ are nondecreasing in $y$ for the fixed $x$, respectively.

A function $x(t)$ is said to be a solution of $(1.1)$, if $x(t)$ is continuous on [0,1] satisfying (1.1) and its corresponding boundary value conditions, and $x^{\prime}$ is continuous on $(0,1), p(t) x^{\prime}$ is twice continuously differentiable on $[0,1]$. 
The paper is organized as follows. We give some necessary lemmas in Section 2 which are main tools to prove the theorems. Then we establish the main results. In Section 3, the multiplicity of the solutions is concerned by using topological degree theory. Also, there are some examples to illustrate how the results of this paper can be applied.

\section{Nagumo theorem}

Lemma 2.1 Let $\psi, \phi$ be the upper and lower solutions of (1.1), respectively, and suppose that Hypotheses 1.1 and 1.2 hold. Then for any solution $x(t)$ of boundary value problem (1.1) with $p(t) \phi^{\prime} \leq p(t) x^{\prime} \leq p(t) \psi^{\prime}, \forall t \in[0,1]$, there always exists $L>0$, such that $\left|\left(p(t) x^{\prime}\right)^{\prime}\right|<L$, $\forall t \in[0,1]$.

Proof Let

$$
\mu_{0}=\int_{0}^{1} A_{2}(t, x) d t, \quad \sigma=\max \left\{p(t) \psi^{\prime}(t)-p(s) \phi^{\prime}(s) ; t, s \in[0,1]\right\} .
$$

There exists $t_{0} \in[0,1]$, such that $\left|\left(p(t) x^{\prime}(t)\right)^{\prime}\right|_{t=t_{0}}<\sigma+1$ by the mean value theorem. Suppose not. Then we have $\sigma_{1}$ and $N_{1}$ satisfying

$$
\sigma_{1}, N_{1}>n+(\sigma+1), \quad \int_{\sigma_{1}}^{N_{1}} h^{-1}(s) d s>(\sigma+1) r+N \mu_{0}
$$

such that $\left(p(t) x^{\prime}\right)^{\prime}$ keeps the same sign on $\left[t_{1}, t_{2}\right] \subset[0,1]$ and $\left.\left(p(t) x^{\prime}\right)^{\prime}\right|_{t_{1}}=\sigma_{1},\left.\left(p(t) x^{\prime}\right)^{\prime}\right|_{t_{2}}=$ $N_{1}$, or inversely. Let the former hold and $\left(p(t) x^{\prime}\right)^{\prime}$ be positive on $\left[t_{1}, t_{2}\right] \subset[0,1]$. Therefore

$$
\begin{aligned}
(\sigma+1) r+N \mu_{0} & <\int_{\sigma_{1}}^{N_{1}} h^{-1}(s) d s \\
& =\int_{t_{1}}^{t_{2}} h^{-1}\left(\left(p(t) x^{\prime}\right)^{\prime}\right)\left|\left(p(t) x^{\prime}\right)^{\prime \prime}\right| d t \\
& \leq \int_{t_{1}}^{t_{2}} A_{1}(t, x)\left(p(t) x^{\prime}\right)^{\prime}+A_{2}(t, x) N d t \\
& <(\sigma+1) r+N \mu_{0},
\end{aligned}
$$

which is a contradiction. The result is concluded.

Next, we consider the auxiliary problem as follows:

$$
\begin{aligned}
& f_{L}\left(t, x, p(t) x^{\prime},\left(p(t) x^{\prime}\right)^{\prime}\right)= \begin{cases}f\left(t, x, p(t) x^{\prime}, L\right), & \left(p(t) x^{\prime}\right)^{\prime}>L, \\
f\left(t, x, p(t) x^{\prime},\left(p(t) x^{\prime}\right)^{\prime}\right), & \left|\left(p(t) x^{\prime}\right)^{\prime}\right| \leq L, \\
f\left(t, x, p(t) x^{\prime},-L\right), & \left(p(t) x^{\prime}\right)^{\prime}<L,\end{cases} \\
& F_{L}\left(t, x, p(t) x^{\prime},\left(p(t) x^{\prime}\right)^{\prime}\right) \\
& \quad= \begin{cases}f_{L}\left(t, x, p(t) \psi^{\prime},\left(p(t) x^{\prime}\right)^{\prime}\right)+\frac{p(t) x^{\prime}-p(t) \psi^{\prime}}{1+p(t) x^{\prime}-p(t) \psi^{\prime},} & p(t) x^{\prime}>p(t) \psi^{\prime}, \\
f_{L}\left(t, x, p(t) x^{\prime},\left(p(t) x^{\prime}\right)^{\prime}\right), & p(t) \phi \leq p(t) x^{\prime} \leq p(t) \psi^{\prime}, \\
f_{L}\left(t, x, p(t) \phi^{\prime},\left(p(t) x^{\prime}\right)^{\prime}\right)-\frac{p(t) \phi^{\prime}-p(t) x^{\prime}}{1+p(t) \phi^{\prime}-p(t) x^{\prime}}, & p(t) x^{\prime}<p(t) \phi^{\prime},\end{cases}
\end{aligned}
$$




$$
\begin{gathered}
F\left(t, x, p(t) x^{\prime},\left(p(t) x^{\prime}\right)^{\prime}\right)= \begin{cases}F_{L}\left(t, \psi, p(t) x^{\prime},\left(p(t) x^{\prime}\right)^{\prime}\right), & x>\psi, \\
F_{L}\left(t, x, p(t) x^{\prime},\left(p(t) x^{\prime}\right)^{\prime}\right), & \phi \leq x \leq \psi, \\
F_{L}\left(t, \phi, p(t) x^{\prime},\left(p(t) x^{\prime}\right)^{\prime}\right), & \phi>x,\end{cases} \\
\begin{cases}\left(p(t) x^{\prime}\right)^{\prime \prime}=F\left(t, x, p(t) x^{\prime},\left(p(t) x^{\prime}\right)^{\prime}\right), & t \in(0,1), \\
x(0)=\alpha,\left.\quad p(t) x^{\prime}(t)\right|_{t=0}=\alpha^{\prime}, & \left.p(t) x^{\prime}(t)\right|_{t=1}=\beta^{\prime} .\end{cases}
\end{gathered}
$$

Lemma 2.2 Let $\psi, \phi$ be the upper and lower solutions of (1.1), respectively, and suppose that Hypotheses 1.1 and 1.2 hold, $\phi(0) \leq \alpha \leq \psi(0),\left.p(t) \phi^{\prime}(t)\right|_{t=0} \leq \alpha^{\prime} \leq\left. p(t) \psi^{\prime}(t)\right|_{t=0}$, $\left.p(t) \phi^{\prime}(t)\right|_{t=1} \leq \beta^{\prime} \leq\left. p(t) \psi^{\prime}(t)\right|_{t=1}$. Then for any solution $x(t)$ of problem (2.1), we have $\phi \leq$ $x \leq \psi, p(t) \phi^{\prime} \leq p(t) x^{\prime} \leq p(t) \psi^{\prime}$ on $[0,1]$.

Proof First, we prove $p(t) x^{\prime}(t) \leq p(t) \psi^{\prime}(t)$ on $[0,1]$. Assume that our conclusion is not true. Then there exists $\hat{t} \in(0,1)$, such that $p(\hat{t}) x^{\prime}(\hat{t})>p(\hat{t}) \psi^{\prime}(\hat{t})$. Hence, $p(t) x^{\prime}-p(t) \psi^{\prime}$ has a positive maximum at $t_{0} \in(0,1)$. We have

$$
\left.\left(p(t) x^{\prime}\right)^{\prime}\right|_{t=t_{0}}=\left.\left(p(t) \psi^{\prime}\right)^{\prime}\right|_{t=t_{0}},\left.\quad\left(p(t) x^{\prime}\right)^{\prime \prime}\right|_{t=t_{0}} \leq\left.\left(p(t) \psi^{\prime}\right)^{\prime \prime}\right|_{t=t_{0}}
$$

But

$$
\left.\left(p(t) x^{\prime}\right)^{\prime \prime}\right|_{t=t_{0}}= \begin{cases}f_{L}\left(t, \psi, p(t) \psi^{\prime},\left(p(t) x^{\prime}\right)^{\prime}\right)+\left.\frac{p(t) x^{\prime}-p(t) \psi^{\prime}}{1+p(t) x^{\prime}-p(t) \psi^{\prime}}\right|_{t=t_{0}}, & x>\psi, \\ f_{L}\left(t, x, p(t) \psi^{\prime},\left(p(t) x^{\prime}\right)^{\prime}\right)+\left.\frac{p(t) x^{\prime}-p(t) \psi^{\prime}}{1+p(t) x^{\prime}-p(t) \psi^{\prime}}\right|_{t=t_{0}}, & \phi \leq x \leq \psi, \\ f_{L}\left(t, \phi, p(t) \psi^{\prime},\left(p(t) x^{\prime}\right)^{\prime}\right)+\left.\frac{p(t) x^{\prime}-p(t) \psi^{\prime}}{1+p(t) x^{\prime}-p(t) \psi^{\prime}}\right|_{t=t_{0}}, & x \leq \phi .\end{cases}
$$

By the definition of $\psi$ and the monotonicity of $f$, we have

$$
\left.\left(p(t) x^{\prime}\right)^{\prime \prime}\right|_{t=t_{0}}>\left.\left(p(t) \psi^{\prime}\right)^{\prime \prime}\right|_{t=t_{0}},
$$

which is a contradiction. Then $p(t) x^{\prime}(t) \leq p(t) \psi^{\prime}(t), \forall t \in[0,1]$. The case of $p(t) x^{\prime} \geq$ $p(t) \phi^{\prime}(t), \forall t \in[0,1]$ is similar. We get $\phi^{\prime} \leq x^{\prime} \leq \psi^{\prime}, \forall t \in(0,1)$. Notice that $x, \psi, \phi$ are continuous on $[0,1]$ and $\phi(0) \leq \alpha \leq \psi(0)$, we can conclude that $\phi(t) \leq x(t) \leq \psi(t), \forall t \in[0,1]$.

Lemma 2.3 Iff of the boundary value problem (1.1) is continuous and bounded on $[0,1] \times$ $\Omega \times \mathbb{R}^{2}$, problem (1.1) has a solution.

Proof Let

$$
Y=\left\{x \in C[0,1] ; x^{\prime} \in C(0,1), p(t) x^{\prime} \in C^{1}[0,1]\right\} .
$$

We define a norm on $Y$ :

$$
\|x\|=\max _{t \in[0,1]}\{|x(t)|\}+\max _{t \in[0,1]}\left\{\left|p(t) x^{\prime}(t)\right|\right\}+\max _{t \in[0,1]}\left\{\left|\left(p(t) x^{\prime}(t)\right)^{\prime}\right|\right\}, \quad x \in Y .
$$

Under this norm, $Y$ is a Banach space.

Let

$$
\begin{aligned}
X= & \left\{x \in C[0,1] ; x^{\prime} \in C(0,1), p(t) x^{\prime} \in C^{2}[0,1], x(0)=\alpha,\left.p(t) x^{\prime}(t)\right|_{t=0}=\alpha^{\prime},\right. \\
& \left.\left.p(t) x^{\prime}(t)\right|_{t=1}=\beta^{\prime}\right\} .
\end{aligned}
$$

We see that $X$ is a convex subset of $Y$. 
Define a mapping $T: X \rightarrow X$ by

$$
\begin{aligned}
{[T x](t)=} & \int_{0}^{t} \int_{0}^{s} \int_{0}^{\tau} p^{-1}(s) f\left(\sigma, x, p x^{\prime},\left(p x^{\prime}\right)^{\prime}\right) d \sigma d \tau d s \\
& +\delta+\lambda \int_{0}^{t} p^{-1}(s) d s+\omega \int_{0}^{t} p^{-1}(s) s d s,
\end{aligned}
$$

where

$$
\begin{aligned}
& \delta=\alpha, \quad \lambda=\left.p(t) x^{\prime}(t)\right|_{t=0}, \\
& \omega=\left.p(t) x^{\prime}(t)\right|_{t=1}-\lambda-\int_{0}^{1} \int_{0}^{\tau} f\left(\sigma, x, p(\sigma) x^{\prime},\left(p(\sigma) x^{\prime}\right)^{\prime}\right) d \sigma d \tau .
\end{aligned}
$$

Then $T(X) \subset X$.

Now, for any $t_{1}, t_{2} \in[0,1]$, we have

$$
\begin{aligned}
& \left|[T x]\left(t_{1}\right)-[T x]\left(t_{2}\right)\right| \\
& \quad=\left|\int_{t_{2}}^{t_{1}} \int_{0}^{s} \int_{0}^{\tau} p^{-1}(s) f\left(\sigma, x, p x^{\prime},\left(p x^{\prime}\right)^{\prime}\right) d \sigma d \tau d s+\lambda \int_{t_{2}}^{t_{1}} p^{-1}(s) d s+\omega \int_{t_{2}}^{t_{1}} p^{-1}(s) s d s\right| \\
& \left|p\left(t_{1}\right)[T x]^{\prime}\left(t_{1}\right)-p\left(t_{2}\right)[T x]^{\prime}\left(t_{2}\right)\right|=\left|\omega\left(t_{1}-t_{2}\right)+\int_{t_{2}}^{t_{2}} \int_{0}^{\tau} f\left(\sigma, x, p(\sigma) x^{\prime},\left(p(\sigma) x^{\prime}\right)^{\prime}\right) d \sigma d \tau\right| \\
& \left|\left(p\left(t_{1}\right)[T x]^{\prime}\left(t_{1}\right)\right)^{\prime}-\left(p\left(t_{2}\right)[T x]^{\prime}\left(t_{2}\right)\right)^{\prime}\right|=\left|\int_{t_{2}}^{t_{1}} f\left(\sigma, x, p(\sigma) x^{\prime},\left(p(\sigma) x^{\prime}\right)^{\prime}\right) d \sigma\right|
\end{aligned}
$$

The function $f$ is bounded. We see that $T(X)$ is bounded and equicontinuous and hence relatively compact in $X$. Let $x_{k}$ be a sequence which converges in $X$ uniformly to $z \in X$. Then $f\left(t, x_{k}, p x_{k}^{\prime},\left(p x_{k}^{\prime}\right)^{\prime}\right) \rightarrow f\left(t, z, p z^{\prime},\left(p z^{\prime}\right)^{\prime}\right)$ in $[0,1]$ because $f$ is uniformly continuous in bounded subsets of $[0,1] \times \Omega \times \mathbb{R}^{2}$. Therefore, $T x_{k} \rightarrow T z, k \rightarrow \infty$, which shows that $T$ is continuous. By the Schauder fixed point theorem, $T$ has at least one fixed point $x(t)$ on $X$. Then $x(t)$ is the desired solution.

Now, we prove our main results.

Theorem 2.1 Let $\psi, \phi$ be the upper and lower solutions of $(1.1)$ on $[0,1]$, respectively, and suppose that Hypotheses 1.1 and 1.2 hold. Then, for any $\alpha, \alpha^{\prime}, \beta^{\prime}$ with $\phi(0) \leq \alpha \leq \psi(0)$, $\left.p(t) \phi^{\prime}(t)\right|_{t=0} \leq \alpha^{\prime} \leq\left. p(t) \psi^{\prime}(t)\right|_{t=0},\left.p(t) \phi^{\prime}(t)\right|_{t=1} \leq \beta^{\prime} \leq\left. p(t) \psi^{\prime}(t)\right|_{t=1}$, the boundary value problem (1.2) has a solution $x(t)$ with $\phi \leq x \leq \psi, p(t) \phi^{\prime} \leq p(t) x^{\prime} \leq p(t) \psi^{\prime}$ on $[0,1]$.

Proof By Lemmas 2.1 and 2.2, the solution of problem (1.2) is also the solution of problem (2.1). Furthermore, $F$ of problem (2.1) is bounded and continuous. There exists a solution $x(t)$ of problem (2.1) by Lemma 2.3. $x(t)$ is also the solution of problem (1.2). This completes the proof.

Theorem 2.2 Let $\psi, \phi$ be the upper and lower solutions of $(1.1)$ on $[0,1]$, respectively, and suppose that Hypotheses 1.1 and 1.2 hold. Then for any $\alpha, \beta^{\prime}, g$ with $\phi(0) \leq \alpha \leq \psi(0)$, $\left.p(t) \phi^{\prime}(t)\right|_{t=1} \leq \beta^{\prime} \leq\left. p(t) \psi^{\prime}(t)\right|_{t=1},\left.g\left(p(t) \phi^{\prime},\left(p(t) \phi^{\prime}\right)^{\prime}\right)\right|_{t=0} \geq 0 \geq\left. g\left(p(t) \psi^{\prime},\left(p(t) \psi^{\prime}\right)^{\prime}\right)\right|_{t=0}$, the 
boundary value problem (1.3) has a solution $x(t)$ with $\phi \leq x \leq \psi, p(t) \phi^{\prime} \leq p(t) x^{\prime} \leq p(t) \psi^{\prime}$ on $[0,1]$.

Proof If we can prove that for any $\epsilon>0, \phi(0) \leq \alpha \leq \psi(0),\left.p(t) \phi^{\prime}(t)\right|_{t=1} \leq \beta^{\prime} \leq\left. p(t) \psi^{\prime}(t)\right|_{t=1}$, problem (1.3) has a solution $x(t, \epsilon)$ on [0,1] satisfying $x(0, \epsilon)=\alpha,\left.p(t) x^{\prime}(t, \epsilon)\right|_{t=1}=\beta^{\prime}$, $\left|g\left(p(t) x^{\prime}(t, \epsilon),\left(p(t) x^{\prime}(t, \epsilon)\right)^{\prime}\right)\right|_{t=0}<\epsilon$, and $\phi \leq x(t, \epsilon) \leq \psi, p(t) \phi^{\prime} \leq p(t) x(t, \epsilon)^{\prime} \leq p(t) \psi^{\prime}$ on $[0,1]$, the result is concluded.

We shall prove the modified theorem. For the fixed $\alpha$, $\beta^{\prime}$ with $\phi(0) \leq \alpha \leq \psi(0)$, $\left.p(t) \phi^{\prime}(t)\right|_{t=1} \leq \beta^{\prime} \leq\left. p(t) \psi^{\prime}(t)\right|_{t=1}$, let

$$
\begin{aligned}
\Pi\left(\alpha^{\prime}\right)= & \left\{x \in C([0,1] ; \Omega) ;\left(p(t) x^{\prime}\right)^{\prime \prime}=f\left(t, x, p(t) x^{\prime},\left(p(t) x^{\prime}\right)^{\prime}\right), p(t) x^{\prime} \in C^{2}[0,1],\right. \\
& x(0)=\alpha,\left.p(t) x^{\prime}(t)\right|_{t=0}=\alpha^{\prime},\left.p(t) x^{\prime}(t)\right|_{t=1}=\beta^{\prime}, x^{\prime} \in C(0,1), \phi \leq x \leq \psi, \\
& \left.p(t) \phi^{\prime} \leq p(t) x^{\prime} \leq p(t) \psi^{\prime}, \forall t \in[0,1]\right\} .
\end{aligned}
$$

By Theorem 2.1, $\Pi\left(\alpha^{\prime}\right)$ is not empty for all $\left.p(t) \phi^{\prime}(t)\right|_{t=0} \leq \alpha^{\prime} \leq\left. p(t) \psi^{\prime}(t)\right|_{t=0}$. Assume that the modified theorem is not true. There exists $\epsilon_{0}>0$, such that

$$
\begin{aligned}
& \left|g\left(p(t) x^{\prime}\left(t, \epsilon_{0}\right),\left(p(t) x^{\prime}\left(t, \epsilon_{0}\right)\right)^{\prime}\right)\right|_{t=0} \geq \epsilon_{0}, \\
& \quad \text { for all }\left.p(t) \phi^{\prime}(t)\right|_{t=0} \leq \alpha^{\prime} \leq\left. p(t) \psi^{\prime}(t)\right|_{t=0}, x(t) \in \Pi\left(\alpha^{\prime}\right) .
\end{aligned}
$$

It is easy to prove that

$$
\left.\left(p(t) x^{\prime}\right)^{\prime}\right|_{t=0} \leq\left.\left(p(t) \psi^{\prime}\right)^{\prime}\right|_{t=0}, \quad \text { for all } x(t) \in \Pi\left(\left.p(t) \psi^{\prime}(t)\right|_{t=0}\right) .
$$

By the monotonicity of $g$, we have

$$
\left.g\left(p(t) x^{\prime},\left(p(t) x^{\prime}\right)^{\prime}\right)\right|_{t=0} \leq\left. g\left(p(t) \psi^{\prime},\left(p(t) \psi^{\prime}\right)^{\prime}\right)\right|_{t=0} \leq-\epsilon_{0} .
$$

The case of $\left.g\left(p(t) \phi^{\prime},\left(p(t) \phi^{\prime}\right)^{\prime}\right)\right|_{t=0} \geq \epsilon_{0}$ for $x(t) \in \Pi\left(\left.p(t) \phi^{\prime}(t)\right|_{t=0}\right)$ is similar.

Let

$$
\begin{aligned}
& C=\left\{x(t) \in \Pi\left(\alpha^{\prime}\right) ;\left.p(t) \phi^{\prime}(t)\right|_{t=0} \leq \alpha^{\prime} \leq\left. p(t) \psi^{\prime}(t)\right|_{t=0},\left.g\left(p(t) \phi^{\prime},\left(p(t) \phi^{\prime}\right)^{\prime}\right)\right|_{t=0} \geq \epsilon_{0}\right\} \\
& \alpha_{0}^{\prime}=\sup \left\{\left.p(t) x^{\prime}(t)\right|_{t=0} ; x(t) \in C\right\} .
\end{aligned}
$$

Obviously, we have $\alpha_{0}^{\prime}<\left.p(t) \psi^{\prime}(t)\right|_{t=0}$. Assume that $x_{0}(t)$ is the solution of (1.1) satisfying $\left.g\left(p(t) x_{0}^{\prime},\left(p(t) x_{0}^{\prime}\right)^{\prime}\right)\right|_{t=0} \geq \epsilon_{0}, x_{0}(0)=\alpha,\left.p(t) x_{0}^{\prime}(t)\right|_{t=0}=\alpha_{0}^{\prime},\left.p(t) x_{0}^{\prime}(t)\right|_{t=1}=\beta^{\prime}$. There exists $N>$ 1 , such that $\alpha_{0}^{\prime}+1 / N<\left.p(t) \psi^{\prime}(t)\right|_{t=0}$. Choose $x_{n} \in \Pi\left(\alpha_{0}^{\prime}+1 / n\right)$ satisfying $p(t) x_{n}^{\prime}(t) \geq p(t) x_{0}^{\prime}(t)$ on $[0,1], \forall n \geq N$. Then $x_{n} \rightarrow \hat{x} \in \Pi\left(\alpha_{0}^{\prime}\right)$ and $p(t) \hat{x}^{\prime} \geq p(t) x_{0}^{\prime}$ on $[0,1]$. By the definition of $\alpha_{0}^{\prime}$, we have $\left.g\left(p(t) x_{n}^{\prime},\left(p(t) x_{n}^{\prime}\right)^{\prime}\right)\right|_{t=0} \leq-\epsilon_{0}$. Hence,

$$
\left.g\left(p(t) \hat{x}^{\prime},\left(p(t) \hat{x}^{\prime}\right)^{\prime}\right)\right|_{t=0} \leq-\epsilon_{0} .
$$

We have

$$
\left.\left(p(t) \hat{x}^{\prime}\right)^{\prime}\right|_{t=0}<\left.\left(p(t) x_{0}^{\prime}\right)^{\prime}\right|_{t=0}
$$


which contradicts $p(t) \hat{x}^{\prime}(t) \geq p(t) x_{0}^{\prime}(t), \forall t \in[0,1],\left.p(t) \hat{x}^{\prime}(t)\right|_{t=0}=\left.p(t) x_{0}^{\prime}(t)\right|_{t=0}=\alpha_{0}^{\prime}$. This completes the proof.

In the same way, we can get Theorem 2.3.

Theorem 2.3 Let $\psi, \phi$ be the upper and lower solutions of $(1.1)$ on $[0,1]$, respectively, and suppose that Hypotheses 1.1 and 1.2 hold. Then, for any $\alpha, \alpha^{\prime}, h$ with $\phi(0) \leq \alpha \leq \psi(0)$, $\left.p(t) \phi^{\prime}(t)\right|_{t=0} \leq \alpha^{\prime} \leq\left. p(t) \psi^{\prime}(t)\right|_{t=0},\left.h\left(p(t) \phi^{\prime},\left(p(t) \phi^{\prime}\right)^{\prime}\right)\right|_{t=1} \leq 0 \leq\left. h\left(p(t) \psi^{\prime},\left(p(t) \psi^{\prime}\right)^{\prime}\right)\right|_{t=1}$, the boundary value problem (1.4) has a solution $x(t)$ with $\phi \leq x \leq \psi, p(t) \phi^{\prime} \leq p(t) x^{\prime} \leq p(t) \psi^{\prime}$ on $[0,1]$.

In the following, we prove our main theorem.

Theorem 2.4 Let $\psi, \phi$ be the upper and lower solutions of (1.1), respectively, and suppose that Hypotheses 1.1 and 1.2 hold. Then, for any $g(x, y), h(x, y), \alpha$ with $\phi(0) \leq$ $\alpha \leq \psi(0),\left.g\left(p(t) \phi^{\prime},\left(p(t) \phi^{\prime}\right)^{\prime}\right)\right|_{t=0} \geq 0 \geq\left. g\left(p(t) \psi^{\prime},\left(p(t) \psi^{\prime}\right)^{\prime}\right)\right|_{t=0},\left.h\left(p(t) \phi^{\prime},\left(p(t) \phi^{\prime}\right)^{\prime}\right)\right|_{t=1} \leq 0 \leq$ $\left.h\left(p(t) \psi^{\prime},\left(p(t) \psi^{\prime}\right)^{\prime}\right)\right|_{t=1}$, the boundary value problem (1.5) has a solution $x(t)$ with $\phi \leq x \leq \psi$, $p(t) \phi^{\prime} \leq p(t) x^{\prime} \leq p(t) \psi^{\prime}$ on $[0,1]$.

Proof For any $\beta^{\prime}$ with $\left.p(t) \phi^{\prime}(t)\right|_{t=1} \leq \beta^{\prime} \leq\left. p(t) \psi^{\prime}(t)\right|_{t=1}$, let

$$
\begin{aligned}
\Pi\left(\beta^{\prime}\right)= & \left\{x \in C[0,1] ; x^{\prime} \in C(0,1),\left(p(t) x^{\prime}\right)^{\prime \prime}=f\left(t, x, p(t) x^{\prime},\left(p(t) x^{\prime}\right)^{\prime}\right), p(t) x^{\prime} \in C^{2}[0,1],\right. \\
& \left.g\left(p(t) x^{\prime},\left(p(t) x^{\prime}\right)^{\prime}\right)\right|_{t=0}=0, x(0)=\alpha,\left.p(t) x^{\prime}(t)\right|_{t=1}=\beta^{\prime}, \phi(0) \leq \alpha \leq \psi(0), \\
& \left.\phi \leq x \leq \psi, p(t) \phi^{\prime} \leq p(t) x^{\prime} \leq p(t) \psi^{\prime}, \forall t \in[0,1]\right\} .
\end{aligned}
$$

Suppose not, there exists $\left.h\left(p(t) x^{\prime},\left(p(t) x^{\prime}\right)^{\prime}\right)\right|_{t=1} \neq 0, \forall x \in \Pi\left(\beta^{\prime}\right)$. We easily get

$$
\begin{aligned}
& \left.h\left(p(t) x^{\prime},\left(p(t) x^{\prime}\right)^{\prime}\right)\right|_{t=1}<0, \quad \forall x \in \Pi\left(\left.p(t) \phi^{\prime}(t)\right|_{t=1}\right), \\
& \left.h\left(p(t) x^{\prime},\left(p(t) x^{\prime}\right)^{\prime}\right)\right|_{t=1}>0, \quad \forall x \in \Pi\left(\left.p(t) \psi^{\prime}(t)\right|_{t=1}\right) .
\end{aligned}
$$

Let

$$
\begin{aligned}
\beta_{0}^{\prime}= & \sup \left\{\left.p(t) x^{\prime}(t)\right|_{t=1} ; x(t) \in \Pi\left(\beta^{\prime}\right),\left.p(t) \phi^{\prime}(t)\right|_{t=1} \leq \beta^{\prime} \leq\left. p(t) \psi^{\prime}(t)\right|_{t=1},\right. \\
& \left.\left.h\left(p(t) x^{\prime},\left(p(t) x^{\prime}\right)\right)^{\prime}\right|_{t=1}<0\right\} .
\end{aligned}
$$

Then $\beta_{0}^{\prime}<\left.p(t) \psi^{\prime}(t)\right|_{t=1}$. By Theorem 2.2, there exists a solution $x_{0}(t)$ of the function (1.1) satisfying $\phi(t) \leq x_{0}(t) \leq \psi(t), p(t) \phi^{\prime}(t) \leq p(t) x_{0}^{\prime}(t) \leq p(t) \psi^{\prime}(t)$ on [0,1], and $\left.g\left(p(t) x_{0}^{\prime},\left(p(t) x_{0}^{\prime}\right)^{\prime}\right)\right|_{t=0}=0,\left.h\left(p(t) x_{0}^{\prime},\left(p(t) x_{0}^{\prime}\right)^{\prime}\right)\right|_{t=1}<0,\left.p(t) x_{0}^{\prime}(t)\right|_{t=1}=\beta_{0}^{\prime}, x_{0}(0)=\alpha$. We replace $\phi(t)$ by $x_{0}(t)$ in Theorem 2.2. Then

$$
\begin{aligned}
D= & \left\{x(t) ; x(t) \in \Pi\left(\beta^{\prime}\right), \beta_{0}^{\prime} \leq \beta^{\prime} \leq\left. p(t) \psi^{\prime}(t)\right|_{t=1}, x_{0} \leq x \leq \psi,\right. \\
& \left.p(t) x_{0}^{\prime} \leq p(t) x^{\prime} \leq p(t) \psi^{\prime}, t \in[0,1]\right\},
\end{aligned}
$$

is not empty. For $x(t) \in D$, we have

$$
\left.h\left(p(t) x^{\prime},\left(p(t) x^{\prime}\right)^{\prime}\right)\right|_{t=1}>0, \quad \inf _{x(t) \in D}\left\{\left.p(t) x^{\prime}(t)\right|_{t=1}\right\}=\beta_{0}^{\prime} .
$$


There exists $N_{1}>1$, such that $\beta_{0}^{\prime}+1 / N_{1}<\left.p(t) \psi^{\prime}(t)\right|_{t=1}$. Now, choose $x_{n} \in \Pi\left(\beta_{0}^{\prime}+1 / n\right)$ satisfying $p(t) x_{n}^{\prime} \geq p(t) x_{0}^{\prime}, \forall n \geq N_{1}$. Then $x_{n} \rightarrow \hat{x} \in \Pi\left(\beta_{0}^{\prime}\right), p(t) \hat{x}^{\prime} \geq p(t) x_{0}^{\prime}$ on $[0,1]$ and $\left.h\left(p(t) x_{n}^{\prime},\left(p(t) x_{n}^{\prime}\right)^{\prime}\right)\right|_{t=1}>0$. Hence,

$$
\left.h\left(p(t) \hat{x}^{\prime},\left(p(t) \hat{x}^{\prime}\right)^{\prime}\right)\right|_{t=1} \geq 0 .
$$

By the monotonicity of $h$ and $\left.h\left(p(t) x_{0}^{\prime},\left(p(t) x_{0}^{\prime}\right)^{\prime}\right)\right|_{t=1}<0$, we have

$$
\left.\left(p(t) x_{0}^{\prime}\right)^{\prime}\right|_{t=1}<\left.\left(p(t) \hat{x}^{\prime}\right)^{\prime}\right|_{t=1}
$$

which contradicts $p(t) \hat{x}^{\prime} \geq p(t) x_{0}^{\prime}, \forall t \in[0,1],\left.p(t) \hat{x}^{\prime}(t)\right|_{t=1}=\left.p(t) x_{0}^{\prime}(t)\right|_{t=1}=\beta_{0}^{\prime}$. This completes the proof.

Here, we give an example to show how the results are applied.

Consider the following boundary value problem:

$$
\left\{\begin{array}{l}
\left(p(t) x^{\prime}\right)^{\prime \prime}=f\left(t, x, p(t) x^{\prime},\left(p(t) x^{\prime}\right)^{\prime}\right), \quad t \in(0,1), \\
x(0)=0,\left.\quad g\left(p(t) x^{\prime},\left(p(t) x^{\prime}\right)^{\prime}\right)\right|_{t=0}=0,\left.\quad h\left(p(t) x^{\prime},\left(p(t) x^{\prime}\right)^{\prime}\right)\right|_{t=1}=0
\end{array}\right.
$$

where $g(x, y)=x, h(x, y)=x+y, p(t)=t^{\frac{1}{2}}, f(t, x, y, z)=x+\sin y+z$.

Let $\psi(t)=1, \phi(t)=-\frac{2}{3} t^{\frac{3}{2}}$. Then

$$
\begin{aligned}
& \left(\left(t^{\frac{1}{2}}\right)\left(-\frac{2}{3} t^{\frac{3}{2}}\right)^{\prime}\right)^{\prime \prime}=0 \geq-\frac{2}{3} t^{\frac{3}{2}}+\sin (-t)+(-1), \\
& \left(t^{\frac{1}{2}}(1)^{\prime}\right)^{\prime \prime}=0 \leq 1, \quad \forall t \in(0,1) .
\end{aligned}
$$

Namely, $\psi(t), \phi(t)$ are the upper and lower solutions of problem (2.2).

Obviously, the function $f$ of problem (2.2) is continuous on $[0,1] \times \Omega \times \mathbb{R}^{2}$ satisfying $|f| \leq A_{1} K_{1}+A_{2} K_{2}$, where $A_{1}(t, x)=1, A_{2}(t, x)=1, K_{1}(t, x, y, z)=z, K_{2}(t, x, y, z)=x+\sin y$. Let $r=\sup \left\{A_{1}(t, x)\right\}=1, h(t)=t+1$. Therefore, Hypotheses 1.1 and 1.2 hold. Moreover, $g(x, y)$, $h(x, y)$ are nondecreasing in $y$ for the fixed $x$ and

$$
\left.g\left(p(t) \phi^{\prime}(t),\left(p(t) \phi^{\prime}(t)\right)^{\prime}\right)\right|_{t=0} \geq 0 \geq\left. g\left(p(t) \psi^{\prime}(t),\left(p(t) \psi^{\prime}(t)\right)^{\prime}\right)\right|_{t=0}
$$

and

$$
\left.h\left(p(t) \phi^{\prime}(t),\left(p(t) \phi^{\prime}(t)\right)^{\prime}\right)\right|_{t=1} \leq 0 \leq\left. h\left(p(t) \psi^{\prime}(t),\left(p(t) \psi^{\prime}(t)\right)^{\prime}\right)\right|_{t=1}
$$

hold. There exists a solution for the boundary value problem (2.2) by Theorem 2.4.

\section{Multiplicity of the solutions}

In this section, we study the multiplicity of the solutions by using topological degree theory. First, assume that $\bar{\psi}, \bar{\phi}$ are strict upper and lower solutions of (1.1) with

$$
\begin{aligned}
& \left(p(t) \bar{\phi}^{\prime}\right)^{\prime \prime}>f\left(t, \bar{\phi}, p(t) \bar{\phi}^{\prime},\left(p(t) \bar{\phi}^{\prime}\right)^{\prime}\right), \\
& \left(p(t) \bar{\psi}^{\prime}\right)^{\prime \prime}<f\left(t, \bar{\psi}, p(t) \bar{\psi}^{\prime},\left(p(t) \bar{\psi}^{\prime}\right)^{\prime}\right), \quad \text { on }(0,1),
\end{aligned}
$$




$$
\begin{aligned}
& \bar{\phi}(t)<\bar{\psi}(t), \quad p(t) \bar{\phi}^{\prime}(t)<p(t) \bar{\psi}^{\prime}(t), \quad \text { for } t=0 \quad \text { and } \\
& p(t) \bar{\phi}^{\prime}(t)<p(t) \bar{\psi}^{\prime}(t), \quad \text { for } t=1 .
\end{aligned}
$$

Similar to (2.1), we consider the auxiliary problem

$$
\begin{cases}\left(p(t) x^{\prime}\right)^{\prime \prime}=\bar{F}\left(t, x, p(t) x^{\prime},\left(p(t) x^{\prime}\right)^{\prime}\right), & t \in(0,1), \\ x(0)=\alpha,\left.\quad p(t) x^{\prime}(t)\right|_{t=0}=\alpha^{\prime}, & \left.p(t) x^{\prime}(t)\right|_{t=1}=\beta^{\prime},\end{cases}
$$

where $\bar{F}$ is defined associated to the pairs of strict upper and lower solutions $(\bar{\psi}, \bar{\phi})$.

Lemma 3.1 Let $\bar{\psi}, \bar{\phi}$ be the strict upper and lower solutions of (1.1), respectively, and suppose that Hypotheses 1.1 and 1.2 hold, $\bar{\phi}(0)<\alpha<\bar{\psi}(0),\left.p(t) \bar{\phi}^{\prime}(t)\right|_{t=0}<\alpha^{\prime}<\left.p(t) \bar{\psi}^{\prime}(t)\right|_{t=0}$, $\left.p(t) \bar{\phi}^{\prime}(t)\right|_{t=1}<\beta^{\prime}<\left.p(t) \bar{\psi}^{\prime}(t)\right|_{t=1}$. Then for any solution $x(t)$ of problem $(3.1)$, we have $\bar{\phi}<x<$ $\bar{\psi}, p(t) \bar{\phi}^{\prime}<p(t) x^{\prime}<p(t) \bar{\psi}^{\prime}$ on $[0,1]$.

Proof From Lemma 2.2, the solution $x(t)$ of problem (3.1) satisfies $\bar{\phi} \leq x \leq \bar{\psi}, p(t) \bar{\phi}^{\prime} \leq$ $p(t) x^{\prime} \leq p(t) \bar{\psi}^{\prime}$ on $[0,1]$. We will obtain the result by contradiction.

If there is $t_{0} \in(0,1)$ such that $\left.\left(p(t) x^{\prime}-p(t) \bar{\phi}^{\prime}\right)\right|_{t=t_{0}}=\min _{t \in[0,1]}\left\{p(t) x^{\prime}-p(t) \bar{\phi}^{\prime}\right\}=0$. Then

$$
\begin{aligned}
& \left.\left(p(t) x^{\prime}-p(t) \bar{\phi}^{\prime}\right)^{\prime}\right|_{t=t_{0}}=0, \\
& \left.\left(p(t) x^{\prime}-p(t) \bar{\phi}^{\prime}\right)^{\prime \prime}\right|_{t=t_{0}} \geq 0 .
\end{aligned}
$$

On the other hand, by the definition of $\bar{\phi}$, we have

$$
\left.\left(p(t) x^{\prime}\right)^{\prime \prime}\right|_{t=t_{0}}<\left.\left(p(t) \bar{\phi}^{\prime}\right)^{\prime \prime}\right|_{t=t_{0}}
$$

which is a contradiction. Then $p(t) \bar{\phi}^{\prime}<p(t) x^{\prime}, \forall t \in[0,1]$. The case of $p(t) x^{\prime}<p(t) \bar{\psi}^{\prime}$, $\forall t \in[0,1]$ is similar. Note that $\bar{\phi}(0)<x(0)<\bar{\psi}(0)$. Therefore, $\bar{\phi}<x<\bar{\psi}$ on $[0,1]$, which completes the proof.

Next, we only focus on the existence of at least three solutions to the boundary value problem (1.2).

Theorem 3.1 Assume that $\phi_{i}, \psi_{i}, i=1,2$ are lower and upper solutions to (1.1), respectively. $\phi_{2}$ and $\psi_{1}$ are strict lower and upper solutions. For all $t \in[0,1]$, we have $\phi_{1} \leq \phi_{2} \leq \psi_{2}, \phi_{1} \leq$ $\psi_{1} \leq \psi_{2}, p(t) \phi_{1}^{\prime} \leq p(t) \phi_{2}^{\prime} \leq p(t) \psi_{2}^{\prime}, p(t) \phi_{1}^{\prime} \leq p(t) \psi_{1}^{\prime} \leq p(t) \psi_{2}^{\prime}$, and $\left\{t \mid \phi_{2} \geq \psi_{1}\right\} \cup\left\{t \mid p(t) \phi_{2}^{\prime} \geq\right.$ $\left.p(t) \psi_{1}^{\prime}\right\}$ is not empty. Suppose that Hypotheses 1.1 and 1.2 associated to $\phi_{i}, \psi_{i}, i=1,2$ hold. Then, for any $\alpha, \alpha^{\prime}, \beta^{\prime}$ with $\max _{i=1,2}\left\{\phi_{i}(0)\right\} \leq \alpha \leq \min _{i=1,2}\left\{\psi_{i}(0)\right\}, \max _{i=1,2}\left\{\left.p(t) \phi_{i}^{\prime}(t)\right|_{t=0}\right\} \leq$ $\alpha^{\prime} \leq \min _{i=1,2}\left\{\left.p(t) \psi_{i}^{\prime}(t)\right|_{t=0}\right\}, \max _{i=1,2}\left\{\left.p(t) \phi_{i}^{\prime}(t)\right|_{t=1}\right\} \leq \beta^{\prime} \leq \min _{i=1,2}\left\{\left.p(t) \psi_{i}^{\prime}(t)\right|_{t=1}\right\}$, there exist at least three solutions $x_{1}(t), x_{2}(t), x_{3}(t)$ to the boundary value problem (1.2) such that

$$
\begin{aligned}
& \phi_{1} \leq x_{1} \leq \psi_{1}, \quad p(t) \phi_{1}^{\prime} \leq p(t) x^{\prime} \leq p(t) \psi_{1}^{\prime}, \\
& \phi_{2} \leq x_{2} \leq \psi_{2}, \quad p(t) \phi_{2}^{\prime} \leq p(t) x^{\prime} \leq p(t) \psi_{2}^{\prime}, \\
& \phi_{1} \leq x_{3} \leq \psi_{2} \quad \text { and } \\
& \left\{t \in[0,1] \mid \psi_{1} \leq x_{3} \leq \phi_{2}\right\} \cup\left\{t \in[0,1] \mid p(t) \psi_{1}^{\prime} \leq p(t) x_{3}^{\prime} \leq p(t) \phi_{2}^{\prime}\right\} \text { is not empty. }
\end{aligned}
$$


Proof Similar to (2.1), we consider the auxiliary problem

$$
\begin{cases}\left(p(t) x^{\prime}\right)^{\prime \prime}=F_{1}\left(t, x, p(t) x^{\prime},\left(p(t) x^{\prime}\right)^{\prime}\right), & t \in(0,1), \\ x(0)=\alpha,\left.\quad p(t) x^{\prime}(t)\right|_{t=0}=\alpha^{\prime}, & \left.p(t) x^{\prime}(t)\right|_{t=1}=\beta^{\prime},\end{cases}
$$

where $F_{1}$ is defined associated to $\left(\phi_{1}, \psi_{2}\right)$. Therefore, the solution $x$ to problem (3.2) is the fixed point of some operator $T_{1}$, i.e., $\left(I-T_{1}\right) x=0$.

With a similar argument in Lemmas 2.1,2.2, and 2.3, there exist a constant $M>0$ and $\Omega=\{x \mid\|x\|<M\}$ such that $T_{1}(\bar{\Omega}) \subset \Omega$. By the Schauder fixed point theorem, we have

$$
\operatorname{deg}\left(I-T_{1}, \Omega, \theta\right)=1 .
$$

Let

$$
\Omega_{\phi_{2}}=\left\{x \in \Omega \mid x>\phi_{2}, p(t) x^{\prime}>p(t) \phi_{2}^{\prime}\right\}
$$

and

$$
\Omega_{\psi_{1}}=\left\{x \in \Omega \mid x<\psi_{1}, p(t) x^{\prime}<p(t) \psi_{1}^{\prime}\right\} .
$$

By assumption, we have $\bar{\Omega}_{\phi_{2}} \cap \bar{\Omega}_{\psi_{1}}=\emptyset$. Therefore, $\Omega \backslash\left(\bar{\Omega}_{\phi_{2}} \cup \bar{\Omega}_{\psi_{1}}\right)$ is not empty. From Lemma 3.1, there is no solution in $\partial \Omega_{\phi_{2}} \cup \partial \Omega_{\psi_{1}}$.

Hence,

$$
\begin{aligned}
\operatorname{deg}\left(I-T_{1}, \Omega, \theta\right)= & \operatorname{deg}\left(I-T_{1}, \Omega_{\phi_{2}}, \theta\right)+\operatorname{deg}\left(I-T_{1}, \Omega_{\psi_{1}}, \theta\right) \\
& +\operatorname{deg}\left(I-T_{1}, \Omega \backslash\left(\bar{\Omega}_{\phi_{2}} \cup \bar{\Omega}_{\psi_{1}}\right), \theta\right) .
\end{aligned}
$$

If $\operatorname{deg}\left(I-T_{1}, \Omega_{\phi_{2}}, \theta\right)=\operatorname{deg}\left(I-T_{1}, \Omega_{\psi_{1}}, \theta\right)=1$, then $\operatorname{deg}\left(I-T_{1}, \Omega \backslash\left(\bar{\Omega}_{\phi_{2}} \cup \bar{\Omega}_{\psi_{1}}\right), \theta\right)=-1$. The result is obtained by using topological degree theory.

Indeed, similar to (2.1), we consider the auxiliary problem

$$
\begin{cases}\left(p(t) x^{\prime}\right)^{\prime \prime}=F_{2}\left(t, x, p(t) x^{\prime},\left(p(t) x^{\prime}\right)^{\prime}\right), & t \in(0,1), \\ x(0)=\alpha,\left.\quad p(t) x^{\prime}(t)\right|_{t=0}=\alpha^{\prime}, & \left.p(t) x^{\prime}(t)\right|_{t=1}=\beta^{\prime},\end{cases}
$$

where $F_{2}$ is defined associated to $\left(\phi_{2}, \psi_{2}\right)$. Therefore, the solution $x$ to problem (3.3) is the fixed point of some operator $T_{2}$, i.e., $\left(I-T_{2}\right) x=0$.

From Lemma 3.1, we have $x>\phi_{2}, p(t) x^{\prime}>p(t) \phi_{2}^{\prime}$. Hence, $x \in \Omega_{\phi_{2}}$. Note that $F_{1}=F_{2}$ in $\Omega_{\phi_{2}}$. We have

$$
1=\operatorname{deg}\left(I-T_{2}, \Omega, \theta\right)=\operatorname{deg}\left(I-T_{2}, \Omega_{\phi_{2}}, \theta\right)=\operatorname{deg}\left(I-T_{1}, \Omega_{\phi_{2}}, \theta\right) .
$$

Similarly, we get $\operatorname{deg}\left(I-T_{1}, \Omega_{\psi_{1}}, \theta\right)=1$, which completes the proof.

Here, we give an example to show how the above theorem is applied. Note that the boundary point $t=1$ is not essential. Then one can consider the following problem:

$$
\left\{\begin{array}{l}
\left(p(t) x^{\prime}\right)^{\prime \prime}=-p(t) x^{\prime}, \quad t \in(0,1), \\
x(0)=0,\left.\quad p(t) x^{\prime}\right|_{t=0}=0,\left.\quad p(t) x^{\prime}\right|_{t=2 \pi}=0,
\end{array}\right.
$$

where $p(t)=t^{\frac{1}{2}}$. A straightforward calculation shows Hypotheses 1.1 and 1.2 hold. 
On the one hand, we can see this problem has solutions

$$
x(t)=C \int_{0}^{t} s^{-\frac{1}{2}} \sin s d s
$$

for any constant $C$.

On the other hand, $\phi_{1}=M, \psi_{2}=-M$ are upper and lower solutions for $M>0$ large enough.

Define

$$
\psi_{1}(t)=\int_{0}^{t} s^{-\frac{1}{2}}(\lambda \cos \lambda s-\lambda k) d s+C^{\prime}
$$

where $C^{\prime}>0,0<\lambda<1,1-\lambda^{2}<\cos 2 \pi \lambda<1$, and $1-\lambda^{2}<k<\cos 2 \pi \lambda<1$.

We check $\psi_{1}(t)$ is a strict upper solution:

$$
\begin{aligned}
& \psi_{1}(0)=C^{\prime}>0, \\
& \left.p(t) \psi_{1}^{\prime}\right|_{t=0}=\left.(\lambda \cos \lambda t-\lambda k)\right|_{t=0}>0, \\
& \left.p(t) \psi_{1}^{\prime}\right|_{t=2 \pi}=\left.(\lambda \cos \lambda t-\lambda k)\right|_{t=2 \pi}>0, \\
& \left(p(t) \psi_{1}^{\prime}\right)^{\prime \prime}+p(t) \psi_{1}^{\prime}=-\lambda^{3} \cos \lambda t+\lambda \cos \lambda t-\lambda k \leq \lambda\left(1-\lambda^{2}\right)-\lambda k<0 .
\end{aligned}
$$

\section{Define}

$$
\phi_{2}(t)=-\int_{0}^{t} s^{-\frac{1}{2}}(\lambda \cos \lambda s-\lambda k) d s-C^{\prime} .
$$

With a similar calculation, $\phi_{2}(t)$ is a strict lower solution and $\left\{t \mid p(t) \phi_{2}^{\prime} \geq p(t) \psi_{1}^{\prime}\right\}$ is not empty.

\section{Competing interests}

The author declares to have no competing interests.

\section{Acknowledgements}

The author sincerely thanks Professor Yong Li for many useful suggestions. The author also thanks the referees for their comments that improved this paper. This work was supported by NSF of China (NSFC) Grant No. 11401250.

Received: 30 January 2015 Accepted: 24 July 2015 Published online: 08 August 2015

\section{References}

1. Sun, J, Zhang, H: Existence of solutions to third-order m-point boundary-value problem. Electron. J. Differ. Equ. 2008, 125 (2008)

2. Liu, Q, Ume, J, Kang, S: Positive solutions of a singular nonlinear third order two-point boundary value problem. J. Math. Anal. Appl. 326, 589-601 (2007)

3. Agarwal, R, O'Regan, D, Wong, P: Positive Solutions of Differential, Difference, and Integral Equations. Kluwer Academic, Boston (1999)

4. Li, S: Positive solutions of nonlinear singular third-order two-point boundary value problem. J. Math. Anal. Appl. 323, 413-425 (2006)

5. Kelley, W: Some existence theorems for nth-order boundary value problems. J. Differ. Equ. 18, 158-169 (1975)

6. Huang, D, Li, Y: Nagumo theorems of nonlinear singular boundary value problems. Nonlinear Anal. 29, 1365-1372 (1997)

7. Agarwal, R, O'Regan, D, Lakshmikantham, V, Leela, S: An upper and lower solution theory for singular Emden-Fowler equations. Nonlinear Anal. 3, 275-291 (2002)

8. Klaasen, G: Differential inequalities and existence theorems for second and third order boundary value problems. J. Differ. Equ. 10, 529-537 (1971)

9. Yao, Q, Feng, Y: The existence of solution for a third-order two-point boundary value problem. Appl. Math. Lett. 15, 227-232 (2002)

10. Du, Z, Ge, W, Lin, X: A new family of iterative methods based on an exponential model for solving nonlinear equations. J. Math. Anal. Appl. 294, 104-112 (2004) 